\title{
Locked-in syndrome caused by a solitary pontine abscess
}

\author{
MARTIN J. MURPHY, DOUGLAS W. BRENTON, \\ CAROLA. ASCHENBRENER AND JOHN C. VAN GILDER \\ From the Departments of Neurology, Pathology (Neuropathology), and Surgery (Neurosurgery), \\ The University of Iowa College of Medicine, Iowa City, Iowa, USA
}

S U M MAR Y The clinical and pathological findings in a patient with locked-in syndrome caused by a solitary pontine abscess are reported for the first time. Successful treatment of brainstem abscess rests on early and accurate diagnosis.

The localising value of the constellation of signs that constitute the "locked-in" syndrome is well established (Nordgren et al., 1971; Plum and Posner, 1972). Although most cases of locked-in syndrome have had a vascular aetiology (Nordgren et al., 1971; Al-Wardi et al., 1975), recent authors have shown that other pathological processes such as neoplasms, (Cherington et al., 1976; Hawkes and Bryan-Smyth, 1976), presumed brainstem encephalitis (Cherington, 1977), and direct trauma (Britt et al., 1977) involving bilaterally the ventral portion of the pons, can also produce it. We present here a patient in whom this syndrome was caused by a solitary pontine abscess.

\section{Case report}

This 40 year old right handed man was in excellent health until late November 1976 when he developed low grade fever, intermittent slurred speech, and drooping of the right eyelid. Symptomatic improvement followed treatment with tetracycline.

One month later, malaise and low grade fever recurred in association with an oropharyngeal infection presumed to be caused by Candida. An unknown antibiotic was prescribed. Slurring of speech was again noted, associated with unsteadiness when walking. Two days later a tooth extraction was performed. Dysarthria became more pronounced, and the patient complained of severe suboccipital headaches, and developed a right hemiplegia in the course of two days.

Address for reprint requests: Dr M. J. Murphy, Department of Neurology, The University of Iowa Hospitals and Clinics, Iowa City, Iowa 52242, USA.

Accepted 15 May 1979
At another hospital, a nucleid brain scan was normal. Lumbar puncture yielded clear fluid under a pressure of $450 \mathrm{~mm}$ of CSF, with a protein content of $3.1 \mathrm{~g} / \mathrm{l}$, glucose of $1.7 \mathrm{mmol} / 1$, and 250 erythrocytes per $\mathrm{mm}^{3}$.

On admission to the University Hospitals the next day his vital signs were normal and general physical examination was negative except for a scar from previous post-traumatic splenectomy. He was alert and would follow commands and respond appropriately to questions by moving his head and left arm, but he could not speak. There was mild resistance to neck flexion. Cranial nerve examination demonstrated bilateral sixth nerve paresis and bilateral weakness of the face, more marked on the right. There was hypoalgesia in the territory of the maxillary division of the right trigeminal nerve. Jaw jerk and gag reflex were absent. He was unable to protrude his tongue and a flaccid right hemiplegia and left hemiparesis were present. Pain perception was impaired on the left side of the body. Muscle stretch reflexes were hyperactive with sustained bilateral ankle clonus and bilateral extensor plantar responses.

Laboratory evaluation was normal. Computerised tomography of the head demonstrated a questionable area of decreased density in the midpontine area. Lumbar puncture yielded mildly xanthrochromic fluid under a pressure of $165 \mathrm{~mm}$ of CSF. There were 250 leucocytes $\mathrm{mm}^{3}(58 \%$ granulocytes and $42 \%$ mononuclear). Cerebrospinal fluid glucose and protein concentrations were $2.1 \mathrm{mmol} / 1$ and $2.1 \mathrm{~g} / 1$ respectively. Gram stain and India ink preparations were negative. Bacterial, fungal, and mycobacterial cultures were negative. Broad spectrum antibiotic treatment was started. On the second hospital day bilateral hori- 
zontal gaze paresis was noted, more marked to the right. On the third day he became "locked-in", manifested by quadriplegia with intermittent ocular bobbing and decerebrate posturing in response to noxious stimuli. He was alert and could communicate only by opening and closing his eyes. The EEG revealed minimal intermittent bilateral slowing of activity. Vertebral angiography demonstrated a tortuous basilar artery with stretching and displacement of the right superior cerebellar artery to the right, indicative of an avascular brainstem mass. Pneumoencephalography (PEG) revealed enlargement of the anteroposterior pontine diameter, posterior displacement of the fourth ventricle, and kinking of the cerebral aqueduct. Bilateral suboccipital craniectomy was performed. The midline cerebellar vermis was split, and green-white, thick, purulent material was aspirated through the rostral floor of the fourth ventricle. The opening was enlarged, revealing an abscess cavity that was subsequently irrigated with bacitracin solution. Gram stained smears and aerobic and anaerobic cultures of the purulent material were negative. After surgery, the patient was ccmatose with small nonreactive pupils and absent corneal reflexes, but with spontaneous respirations. Withdrawal of the right lower and left upper extremities to pain was present. He progressively deteriorated and died two days after surgery.

\section{PATHOLOGICAL FINDINGS}

Postmortem examination was restricted to the intracranial contents. The brain weighed 1620 grams. Except for parenchymal and meningeal congestion of the cerebrum, pathological findings were confined to the cerebellum and brainstem. A linear abscess cavity measuring up to $8 \mathrm{~mm}$ in width occupied the middle third of the pons throughout its restrocaudal extent. This cavity was filled with creamy purulent exudate and surrounded by a hyperemic margin (Figure). The abscess extended into the fourth ventricle and was coextensive with a large area of haemorrhagic necrosis that involved much of the cerebellar vermis and medial portions of both cerebellar hemispheres. Although the abscess cavity was limited to the pons, the leptomeninges were thickened and opacified over the entire brainstem, and haemorrhagic softening extended rostrally along the ventromedial portions of the midbrain to the diencephalic junction. The diencephalon appeared grossly normal, but there was notable softening of the adenohypophysis.

On histological examination the abscess cavity contained necrotic debris and acute inflammatory exudate. Although marginal hypervascularity and mild gliosis were present, the abscess wall was not well defined, and extensive foci of cerebritis and perivascular inflammation were present throughout the pons and extended anteriorly along the ventromedial portion of the brain. Numerous perivascular haemorrhages and acute inflammatory infiltrates were noted in the midbrain, ventrally and medially to the substantia nigra, but there was no inflammation of the periaqueductal or tectal portions of the midbrain. The medulla was moderately oedematous throughout its extent and the leptomeninges over the entire brainstem were infiltrated by mixed inflammatory cells with plasma cells predominant. Extensive coagulation necrosis was present in the adenohypophysis without significant inflammation. Cultures of the abscess contents grew multiple organisms, Pseudomonas aeruginosa predominating.

\section{Discussion}

Solitary abscesses of the brainstem are infrequent, presenting challenging problems in their diagnosis and treatment (Lichtenstein and Zeitlin, 1936; Weickhardt and Davis, 1964). Until quite recently rapid deterioration and fatal outcome were almost universal. The successful surgical evacuation with survival which has been documented in several recent reports (Danziger et al., 1974; Van Gilder et al., 1974; Robert et al., 1975; Messina et al., 1977) underscores the need for early and accurate diagnosis.

In most cases, the suppurative process results from direct extension from a contiguous focus of infection or haematogenous spread from a distant source, (Weickhardt and Davis, 1964; Van Gilder et al., 1974). In approximately one-third of the patients reported its origin was unknown or could not be determined (Lichtenstein and Zeitlin, 1936; Weickhardt and Davis, 1964), as in the present case.

Headaches, frequently occipital in location, fever, rapidly evolving signs indicating involvement of brainstem structures and spinal fluid pleocytosis are the most characteristic features associated with brainstem abscess, (Weickhardt and Davis, 1964). Resistance to cervical manipulation has been described frequently, but classical meningeal signs are unusual.

Because pontine abscesses tend to involve the tegmentum, most patients present with a combination of ipsilateral cranial nerve signs and contralateral hemiplegia or dissociated contralateral hemianaesthesia, but classical brainstem syndromes that indicate an accurate anatomical localisation 


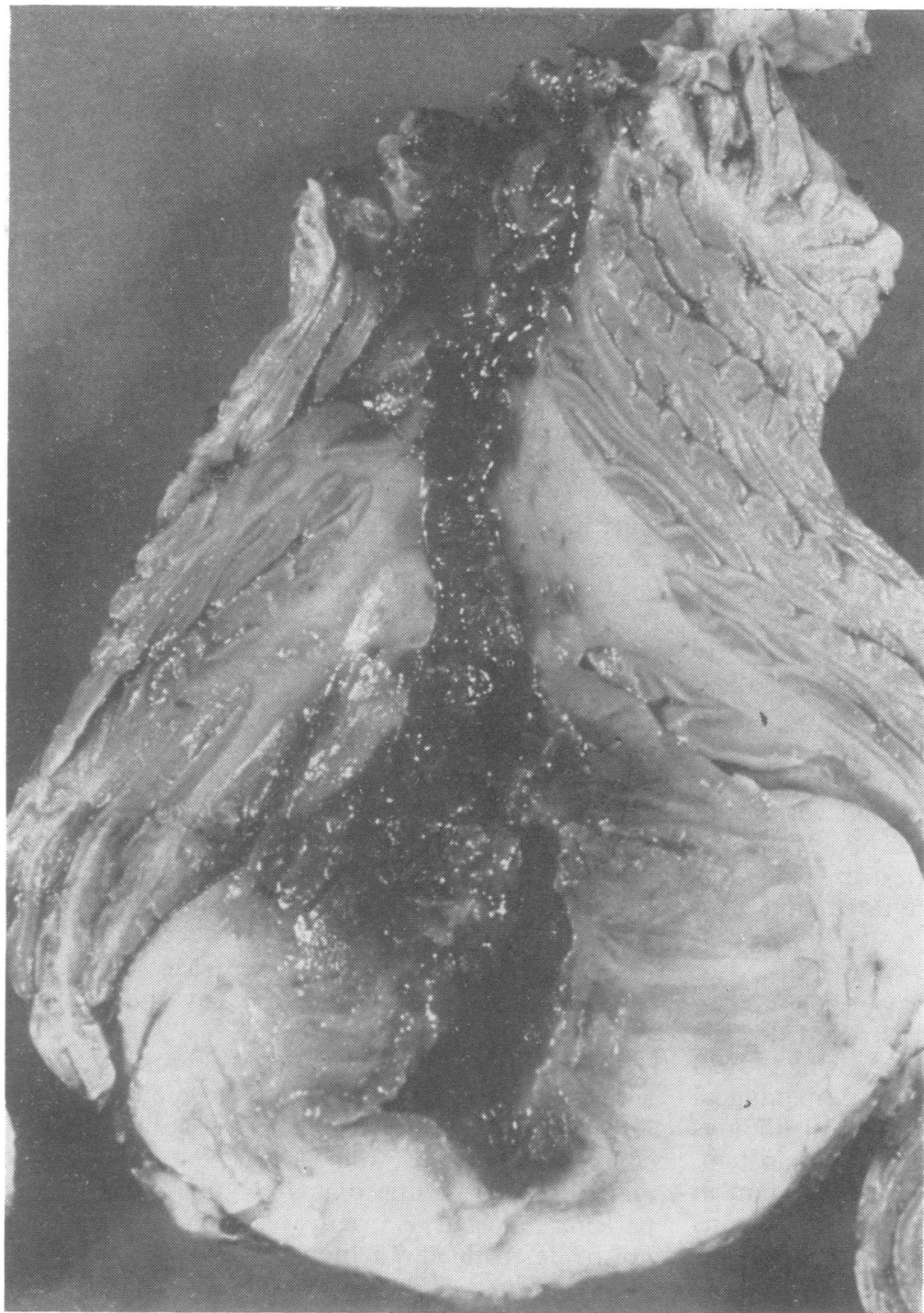

Figure Transverse section through mid-pons and dorsal cerebellum. The central abscess cavity is filled with purulent exudate and has a hyperaemic margin. Dorsally the linear area of haemorrhagic necrosis involving the cerebellum in midline corresponds to the surgical approach.

have been infrequent because of the tendency of the abscess to adopt an elongated tubular shape, involving structures at different levels (Weickhardt and Davis, 1964).

The development of bilateral facial palsy, impaired lateral eye movements, bulbar weakness, anarthria, and quadriparesis with preservation of consciousness rendered our patient "locked-in". As in the cases of locked-in syndrome from other causes, the abscess cavity and surrounding inflammation was located in the ventromedial portion of the pons throughout its rostrocaudal extent, with lesser inflammatory involvement of the ventral portion of the midbrain. Preservation of conscious- ness in these patients is thought to reflect the sparing of reticular structures located in the tegmentum of the pons and midbrain, essential for the maintenance of alertness (Chase et al., 1968).

Although not realised in our patient, the possibility of demonstrating the abscess by computerised tomography of the head reported by Messina et al. (1977) represents a major diagnostic advance.

\section{References}

Al-Wardi, D. A. M., Adams, A. H., and Hamilton, A. E. (1975). Four cases of "locked-in" syndrome and review of the literature. Bulletin of the Los Angeles Neurological Societies, 40, 60-70. 
Britt, R. H., Herrick, M. K., and Hamilton, R. D. (1977). Traumatic locked-in syndrome. Annals of Neurology, 1, 590-592.

Chase, T. N., Moretti, I. L., and Prensky, A. L. (1968). Clinical and electroencephalographic manifestations of vascular lesions of the pons. Neurology (Minneapolis), 18, 357-368.

Cherington, J. (1977). Locked-in syndrome after swine flu inoculation. Archives of Neurology (Chicago), 34, 258.

Cherington, M., Stears, J., and Hodges, J. (1976). Locked-in syndrome caused by a tumor. Neurology (Minneapolis), 26, 180-182.

Danziger, J., Allen, K. L., and Bloch, S. (1974). Brainstem abscess in childhood. Case report. Journal of Neurosurgery, 40, 391-393.

Hawkes, C. H., and Bryan-Smyth, L. (1976). Locked-in syndrome caused by a tumor. Letter to the Editor. Neurology (Minneapolis), 26, 1185.

Lichtenstein, B. W., and Zeitlin, H. (1936). Pontine abscess. Report of two cases. Journal of the American Medical Association, 106, 1057-1060.
Messina, A. V., Guido, L. J., and Liebeskind, A. L. (1977). Preoperative diagnosis of brainstem abscess by computerised tomography with survival. Journal of Neurosurgery, 47, 106-108.

Nordgren, R. E., Markesbery, W. R., Fukuda, K., and Reeves, A. G. (1971). Seven cases of cerebromedullospinal disconnection: the "locked-in" syndrome. Neurology (Minneapolis), 21, 1140-1148.

Plum, F., and Posner, J. B. (1972). Stupor and Coma, second edition. F. A. Davis Company: Philadelphia.

Robert, C. M., Stern, W. E., Brown, W. J., Greenfield, M. A., and Bentson, J. R. (1975). Brainstem abscess treated surgically with special note upon the employment of thorium dioxide. Surgical Neurology, 3, 153-160.

Van Gilder, J. C., Allen, W. E., and Lesser, R. A. (1974). Pontine abscess: survival following surgical drainage. Journal of Neurosurgery, 40, 386-390.

Weickhardt, G. D., and Davis, R. L. (1964). Solitary abscess of the brainstem. Neurology (Minneapolis), 14, 918-925. 